\title{
1 De novo Variants in Neurodevelopmental Disorders with Epilepsy
}

*Henrike O. Heyne1,2,5,26, Tarjinder Singh2,26, Hannah Stamberger21,22,23, Rami Abou Jamra $^{1}$, Hande Caglayan ${ }^{12}$, Dana Craiu13, Peter De Jonghe21,22,23, Renzo Guerrini14, Katherine L. Helbig7, Bobby P. C. Koeleman ${ }^{15}$, Jack A. Kosmicki 2 26, Tarja Linnankivi ${ }^{16}$, Patrick May ${ }^{11}$, Hiltrud Muhle ${ }^{19}$, Rikke S. Møller ${ }^{24,25}$, Bernd A. Neubauer ${ }^{18}$, Aarno Palotie ${ }^{2}$, Manuela Pendziwiat ${ }^{19}$, Pasquale Striano ${ }^{20}$, Sha Tang ${ }^{4}$, Sitao $\mathrm{Wu}^{4}$, EuroEPINOMICS RES Consortium**, Annapurna Poduri6, Yvonne G. Weber ${ }^{8}$, Sarah Weckhuysen ${ }^{21,22,23}$, Sanjay M. Sisodiya ${ }^{9,10}$, Mark Daly ${ }^{2,26}$, Ingo Helbig7,19, Dennis Lal2,3,26, *Johannes R. Lemke ${ }^{1}$

\section{**EuroEPINOMICS RES Consortium}

Zaid Afawi, Carolien de Kovel, Petia Dimova, Tania Djémié, Milda Endziniene, Dorota Hoffman-Zacharska, Johanna Jähn, Christian Korff, Anna-Elina Lehesjoki, Carla Marini, Stefanie H. Müller, Deb Pal, Niklas Schwarz, Kaja Selmer, Jose Serratosa, Ulrich Stephani, Katalin Štěrbová, Arvid Suls, Steffen Syrbe, Inga Talvik, Shan Tang, Sarah von Spiczak, Federico Zara

\section{*corresponding author}

1 University of Leipzig Hospitals and Clinics, Leipzig, Germany

2 Broad Institute, Stanley Center for Psychiatric Research, Cambridge, MA, USA

3 Cologne Center for Genomics (CCG), Cologne, Germany

4 Division of Clinical Genomics, Ambry Genetics, Aliso Viejo, California, USA

5 IFB AdiposityDiseases, University of Leipzig Hospitals and Clinics, Leipzig, Germany

6 Epilepsy Genetics Program, Department of Neurology, Division of Epilepsy and Clinical Neurophysiology, Boston Children's Hospital, Boston, MA, USA

7 Division of Neurology Children's Hospital of Philadelphia, Philadelphia, Pennsylvania, USA

8 Department of Neurology and Epileptology, Hertie Institute for Clinical Brain Research, University of Tübingen, Tübingen, Germany

9 Department of Clinical and Experimental Epilepsy NIHR University College London Hospitals Biomedical Research Centre UCL Institute of Neurology, London, United Kingdom

10 The Epilepsy Society Chalfont-St-Peter Bucks, United Kingdom

11 Luxembourg Centre for Systems Biomedicine, Esch-sur-Alzette, University of Luxembourg, Luxembourg

12 Department of Molecular Biology and Genetics Bogaziçi University Istanbul, Turkey

13 "Carol Davila" University of Medicine Bucharest, Department of Clinical Neurosciences (No.6), Pediatric Neurology Clinic, Alexandru Obregia Hospital, Bucharest, Romania

14 Pediatric Neurology and Neurogenetics Unit and Laboratories A. Meyer Children's Hospital-University of Florence, Florence, Italy

15 Department of Medical Genetics University Medical Center Utrecht, Utrecht, The Netherlands

16 Department of Pediatric Neurology, Children's Hospital, University of Helsinki and Helsinki University Hospital, Helsinki, Finland 17

18 Department of Pediatric Neurology, University Hospital Giessen, Giessen, Germany 19 Department of Neuropediatrics, University Medical Center Schleswig-Holstein, Christian-Albrechts University, Kiel, Germany 
20 Pediatric Neurology and Muscular Diseases Unit, Department of Neurosciences, Rehabilitation, Ophthalmology, Genetics, and Maternal and Child Health, University of Genoa 'G. Gaslini' Institute, Genova, Italy 21 Neurogenetics Group, Center for Molecular Neurology, VIB, Antwerp, Belgium 22 Laboratory of Neurogenetics, Institute Born-Bunge, University of Antwerp, Antwerp, Belgium 23 Division of Neurology, University Hospital Antwerp, Antwerp, Belgium 24 The Danish Epilepsy Centre, Dianalund, Denmark 25 Institute for Regional Health Services, University of Southern Denmark, Odense, Denmark

26 Analytical and Translational Genetics Unit

\section{Acknowledgements}

We like to thank all patients and their families who participated in this study, as well as the teams who were involved in recruiting patients, samples and data at the respective study sites. We thank Lisenka Vissers and Christian Gilissen for epilepsy and age phenotypes from the cohort of Lelieveld et al, 2016 and Jeremy McRae for useful discussions on the DDD cohort (McRae et al, 2016). We thank Johannes Krause for support in figure design and helpful discussions. We are grateful to members of ATGU and the Institute for Human Genetics in Leipzig for helpful contributions. Supported by the Eurocores program EuroEPINOMICS, the Fund for Scientific Research Flanders (FWO), the International Coordination Action (ICA) grant G0E8614N, and the University of Antwerp (research fund). HOH was supported by stipends from the Federal Ministry of Education and Research (BMBF), Germany, FKZ: 01E01501 and the German Research Foundation (DFG): HE7987/1-1. HS is PhD fellow of the Fund for Scientific Research Flanders (1125416N). IH and YGW were supported by DFG grants WE4896/3-1 and HE5415/6-1. RG received funding through the EU 7th Framework Program (FP7) under the project DESIRE grant N602531.

The DDD study presents independent research commissioned by the Health Innovation Challenge Fund [grant number HICF-1009-003], a parallel funding partnership between the Wellcome Trust and the Department of Health, and the Wellcome Trust Sanger Institute [grant number WT098051]. The views expressed in this publication are those of the author(s) and not necessarily those of the Wellcome Trust or the Department of Health. The study has UK Research Ethics Committee approval (10/H0305/83, granted by the Cambridge South REC, and GEN/284/12 granted by the Republic of Ireland REC). The research team acknowledges the support of the National Institute for Health Research, through the Comprehensive Clinical Research Network. 
bioRxiv preprint first posted online Apr. 3, 2017; doi: http://dx.doi.org/10.1101/123323. The copyright holder for this preprint

(which was not peer-reviewed) is the author/funder, who has granted bioRxiv a license to display the preprint in perpetuity.

All rights reserved. No reuse allowed without permission.

\begin{abstract}
93 Abstract
94

Epilepsy is a frequent feature of neurodevelopmental disorders (NDD) but little is

96 known about genetic differences between NDD with and without epilepsy. We analyzed

97 de novo variants (DNV) in 6753 parent-offspring trios ascertained for different NDD. In

98 the subset of 1942 individuals with NDD with epilepsy, we identified 33 genes with a

99 significant excess of DNV, of which SNAP25 and GABRB2 had previously only limited evidence for disease association. Joint analysis of all individuals with NDD also implicated CACNA1E as a novel disease gene. Comparing NDD with and without epilepsy, we found missense DNV, DNV in specific genes, age of recruitment and severity of intellectual disability to be associated with epilepsy. We further demonstrate to what extent our results impact current genetic testing as well as treatment, emphasizing the benefit of accurate genetic diagnosis in NDD with epilepsy.
\end{abstract}




\section{Introduction}

Epilepsies, defined as recurrent, unprovoked seizures, affect about 50 million people worldwide (www.who.int, 03/2017). A significant subset of severe and intractable epilepsies starts in infancy and childhood and poses a major clinical burden to patients, families, and society 1 . Early onset epilepsies are often comorbid with neurodevelopmental disorders (NDD), such as developmental delay, intellectual disability and autism spectrum disorders (DD, ID, ASD) ${ }^{2-4}$, while up to $26 \%$ of individuals with NDD have epilepsy, depending on the severity of intellectual impairment4-6. Several genes have been implicated in both NDD and epilepsy disorders 7,8 . The epileptic encephalopathies (EE) comprise a heterogeneous group of epilepsy syndromes characterized by frequent and intractable seizures that are thought to contribute to developmental regression ${ }^{3,9}$. Phenotypic categorisation of clinicallyrecognizable EE syndromes enabled identification of several associated genes $1,2,10$. However, the phenotypic spectrum of these disease genes was broader than expected ${ }^{11,12}$, ranging from EE (e.g. $S C N 1 A^{13}, K C N Q 2^{14}$ ) to unspecific NDD with or without epilepsy (e.g. $S C N 2 A^{15}$, STXBP116). While clinically distinguishable entities exist, many patients with NDD and epilepsy are not easily classified into EE syndromes ${ }^{1,12}$. Consequently, EE is often used synonymously with NDD with epilepsy ${ }^{17}$. Targeted sequencing of disease-specific gene panels is commonly used in diagnostics of epilepsies $12,18,19$. However, epilepsy gene panel designs of diagnostic laboratories differ substantially in gene content ${ }^{19}$.

Application of a mutational model 18 to detect enrichment for de novo variants (DNV) has proven to be a powerful approach for identification of disease-associated genes in neurodevelopmental disorders including ID, congenital heart disease, schizophrenia and ASD ${ }^{20-23}$. For EE, the currently largest exome-wide DNV burden study comprised 356 parent-offspring trios of two classic EE syndromes (infantile/epileptic spasms, IS and Lennox-Gastaut syndrome, LGS) and revealed seven genes at exomewide significance 24 . To identify genes that are significantly associated with NDD with epilepsy, we analysed 6753 parent-offspring trios of NDD, focusing on 1942 cases with epilepsy including 529 individuals with epileptic encephalopathy. We compared rates of DNV between EE, NDD with unspecified epilepsies and NDD without epilepsy to identify genetic differences between these phenotypic groups. We further investigated the potential impact of our findings on the design of genetic testing approaches and assessed the extent of therapeutically relevant diagnoses. 


\section{Results}

\section{Description of dataset}

We analysed DNV in parent-offspring trios of eight published7,20,23-27, one partly published $^{28}$ and three unpublished cohorts of in total 6753 individuals with NDD stratifying for the 1942 cases with epilepsy (Supplementary Table 1, Figure 1, Online Methods). These 1942 patients were ascertained for either EE or NDD with unspecified epilepsy (DD21, ASD 11 with ID and ID $^{20}$ ). We define those two phenotype groups as $\mathrm{NDD}_{\mathrm{EE}}(\mathrm{n}=529)$ and $\mathrm{NDD}_{\mathrm{uE}}(\mathrm{n}=1413)$, respectively. We later compared $\mathrm{DNV}$ in NDD with epilepsy $\left(\mathrm{NDD}_{\mathrm{EE}+\mathrm{uE}}\right)$ to $\mathrm{DNV}$ in $\mathrm{NDD}$ without epilepsy $\left(\mathrm{NDD}_{\mathrm{woE}}, \mathrm{n}=4811\right)$. For genotype-phenotype comparisons, we restricted our analysis to regions that were adequately captured across different capture solutions (see Online Methods). For ASD data from the Simon Simplex Consortium ${ }^{29}$, we included only individuals with IQ $<70$ (defined as ID) as different studies have found DNV only associated with low-IQ ASD ${ }^{6,30}$. Individuals with $\mathrm{NDD}_{\mathrm{EE}}$ were diagnosed with following specific syndromes: IS ( $\mathrm{n}=243$ ), LGS ( $\mathrm{n}=145$ ), electrical status epilepticus in sleep (ESES, $n=42$ ), myoclonic-atonic epilepsy (MAE, $n=39)$, Dravet syndrome (DS, $n=16)$, unspecified EE $(n=44)$. Six of eight NDD cohorts $(n=6037)$ included individuals with as well as without epilepsy $20,23,25-27,31$. Of these, $20.3 \%$ of patients had epilepsy. In cohorts with more severe ID, a higher rate of patients had epilepsy (Spearman-Rank correlation, $p$-value $=0.012$, rho $=0.89$, Supplementary Figure S2), in line with previous literature ${ }^{4,6}$. We considered DNV of 1911 healthy siblings of patients with ASD as a control group.

\section{$D N V$ in known EE genes in patients with different NDD diagnoses}

We first compared DNV in known EE genes between NDD $\mathrm{EE}_{\mathrm{E}}, \mathrm{NDD}_{\mathrm{uE}}, \mathrm{NDD}_{\mathrm{woE}}$ and control cohorts. We investigated missense and truncating DNV ( $\left.\mathrm{DNV}_{\text {misttrunc }}\right)$ in 50 known autosomal dominant or X-linked EE genes (updated list from ${ }^{19}$, Supplementary Table 3). We excluded DNV present in ExAC ${ }^{32}$ to improve power, as these have been shown to confer no risk to childhood-onset NDD on a group level ${ }^{33}$. The frequency of $\mathrm{DNV}_{\text {mistrunc }}$ in EE genes was not significantly different between $\mathrm{NDD}_{\mathrm{EE}}(13.0 \% \pm 3.1$, mean, $95 \%-\mathrm{CI})$ and $\mathrm{NDD}_{\mathrm{uE}}(11.5 \% \pm 1.8$, mean, 95\%-CI, p-value $=0.4$, Fisher's Exact Test, Figure $1 \mathrm{~A}$, see Supplementary Figure S2 for individual cohorts), but was significantly greater than in $\mathrm{NDD}_{\text {woE }}\left(2.7 \% \pm 0.5\right.$, mean, $95 \%-\mathrm{CI}, \mathrm{p}$-value $\left.=4.4 \times 10^{-46}\right)$ and in healthy controls $(0.3 \% \pm 0.2 \text {, mean, } 95 \%-C I)^{20}$. Within three different NDD diagnoses (ID, ASD [with and without ID], DD), we detected more DNV in EE genes in individuals with epilepsy than without epilepsy (Cochran-Mantel-Haenszel test, p-value 3.5 $\times 10^{-43}$, common OR 4.6, 95\%-CI: 3.7 to 5.9, Figure 2B). This suggests a markedly overlapping genetic spectrum of $\mathrm{NDD}_{\mathrm{EE}}$ and $\mathrm{NDD}_{\mathrm{uE}}$. We subsequently performed $\mathrm{DNV}$ enrichment analyses on the combined cohort of NDD $\mathrm{EE}_{\mathrm{E}+\mathrm{UE}}$.

\section{Discovery of genes with exome-wide DNV burden in NDD with epilepsy}

We compared the numbers of DNV in the combined cohort of NDD with epilepsy $\left(\mathrm{NDD}_{\mathrm{EE}+\mathrm{uE}}\right)$, to the number of $\mathrm{DNV}$ expected by a mutational model ${ }^{30}$ revealing global enrichment of truncating (2.3-fold, $p_{\text {trunc }}=1 \times 10^{-47}$, Poisson Exact test, see Online Methods) and missense (1.6-fold, $\mathrm{p}_{\mathrm{mis}}=2 \times 10^{-33}$ ) but not synonymous DNV ( 0.6 fold, $\left.\mathrm{p}_{\text {syn }}=1.0\right)$. We identified 33 genes with an exome-wide significant burden of $D N V_{\text {mistrunc }}$ (Table 1), of which KCNQ2 $(\mathrm{n}=21), \operatorname{SCN} 2 A(\mathrm{n}=20)$ and $S C N 1 A(\mathrm{n}=19)$ were most frequently mutated. GABRB2 and SNAP25 had previously no statistical evidence for disease association (see Supplementary Note). Beyond the 33 genes with exome-wide significant DNV burden, 114 genes had at least two DNV $_{\text {mistrunc }}$ in our cohort (Supplementary Table 6). After DNV enrichment analysis, we again excluded DNV in ExAC $^{32}$ to improve specificity 33 . 
bioRxiv preprint first posted online Apr. 3, 2017; doi: http://dx.doi.org/10.1101/123323. The copyright holder for this preprint (which was not peer-reviewed) is the author/funder, who has granted bioRxiv a license to display the preprint in perpetuity.

All rights reserved. No reuse allowed without permission.

Collectively analysing all patients with NDD with or without epilepsy ( $\mathrm{n}=6753)$, we found 101 genes with exome-wide DNV burden (Supplementary Table 7). Among these 101 genes five were mutated in at least one individual with EE and at least two other individuals with epilepsy with DNV in the same variant class. Of these, SMARCA2 DYNC1H1 and SLC35A2 were formerly associated with NDD with epilepsy. KCNQ3 had previously limited association with NDD with epilepsy and CACNA1E had previously no statistical evidence for disease association (Genes further described in Supplementary Notes).

Table 1, Genes with exome-wide DNV burden in NDD with epilepsy

\begin{tabular}{|c|c|c|c|c|c|}
\hline \multirow{2}{*}{$\begin{array}{l}\text { Gene } \\
\text { Epilepsy }\end{array}$} & \multicolumn{2}{|c|}{$\mathrm{DNV}_{\text {trunc }}$} & \multicolumn{2}{|c|}{$\mathrm{DNV}_{\text {mis }}$} & \multirow{2}{*}{$\begin{array}{l}\mathrm{DNV}_{\text {misstrunc }} \\
\text { yes+no }\end{array}$} \\
\hline & yes $r$ & no $y$ & yes & no & \\
\hline KCNQ2 & 0 & 1 & 21 & 3 & 25 \\
\hline $\mathrm{SCN} 2 \mathrm{~A}$ & 2 & 12 & 18 & 5 & 37 \\
\hline SCN1A & 8 & 0 & 11 & 0 & 19 \\
\hline CHD2 & 9 & 1 & 3 & 2 & 15 \\
\hline SYNGAP1 & 10 & 7 & 1 & 2 & 20 \\
\hline STXBP1 & 4 & 3 & 7 & 5 & 19 \\
\hline SCN8A & 0 & 1 & 10 & 3 & 14 \\
\hline MEF2C & 4 & 1 & 5 & 0 & 10 \\
\hline SLC6A1 & 2 & 1 & 7 & 3 & 13 \\
\hline DNM1 & 0 & 0 & 9 & 2 & 11 \\
\hline EEF1A2 & 0 & 0 & 8 & 3 & 11 \\
\hline CDKL5 & 2 & 0 & 6 & 0 & 8 \\
\hline DYRK1A & 7 & 9 & 0 & 5 & 21 \\
\hline SMC1A & 7 & 0 & 0 & 2 & 9 \\
\hline GABRB3 & 0 & 0 & 7 & 1 & 8 \\
\hline KIAA2022 & 6 & 0 & 0 & 0 & 6 \\
\hline ASXL3 & 6 & 12 & 0 & 0 & 18 \\
\hline WDR45 & 5 & 5 & 1 & 0 & 11 \\
\hline ARID1B & 6 & 28 & 0 & 2 & 36 \\
\hline GNAO1 & 0 & 1 & 6 & 2 & 9 \\
\hline ALG13 & 0 & 0 & 6 & 0 & 6 \\
\hline $\mathrm{KCNH} 1$ & 0 & 0 & 6 & 2 & 8 \\
\hline GRIN2B & 0 & 3 & 6 & 9 & 18 \\
\hline HNRNPU & 5 & 2 & 0 & 1 & 8 \\
\hline PURA & 3 & 4 & 2 & 4 & 13 \\
\hline GABRB2 & 0 & 0 & 5 & 1 & 6 \\
\hline COL4A3BP & 0 & 0 & 5 & 4 & 9 \\
\hline MECP2 & 2 & 5 & 3 & 5 & 15 \\
\hline FOXG1 & 2 & 3 & 3 & 3 & 11 \\
\hline ANKRD11 & 4 & 28 & 0 & 2 & 34 \\
\hline SNAP25 & 1 & 0 & 3 & 0 & 4 \\
\hline DDX3X & 3 & 19 & 1 & 11 & 34 \\
\hline IQSEC2 & 3 & 2 & 1 & 3 & 9 \\
\hline
\end{tabular}


${ }^{*}$ Genes in order of decreasing numbers of DNV mistrunc in NDD with epilepsy

Phenotypic, biological and therapeutic properties of genes with DNV burden in NDD with epilepsy

We aimed to explore whether the 33 genes with DNV burden in NDD with epilepsy $\left(\mathrm{NDD}_{\mathrm{EE}+\mathrm{uE}}\right)$ were associated with specific phenotypes. Analyses of human phenotype ontology ${ }^{34}$ (HPO) terms revealed most significant enrichment in genes associated with "epileptic encephalopathy" (see Online Methods, Supplementary Table 8). After excluding the 529 patients diagnosed with EE from the DNV enrichment analysis, the most significantly enriched HPO term was still "epileptic encephalopathy" (Bonferroni p-value $3.6 \times 10^{-14}$ ), confirming our previous findings (Figure 1). Per DNV-enriched gene, we plotted distribution of EE phenotypes, sex and seizure phenotypes of generalized, focal, febrile or spasms (Supplementary Figure S6 - 8).

Since the disease onset of NDD with epilepsy is typically in infancy and early childhood, we evaluated expression levels of the 33 genes with DNV burden in the developing infant brain (expression data: brainspan.org, see Online Methods). At a group level, these genes showed high levels of brain expression (Supplementary Figure S9A). The DNV-enriched genes were also substantially depleted for truncating and missense variants in the ExAC control data (Supplementary Figure S9B, S9C). Genes with at least two DNV in $\mathrm{NDD}_{\mathrm{EE}+\mathrm{uE}}$, but no significant DNV burden showed similar patterns.

We finally evaluated if genes with $\mathrm{DNV}_{\text {mistrunc }}$ in NDD with epilepsy were associated with therapy. For each gene, we used criteria from the Centre for EvidenceBased Medicine (CEBM) ${ }^{35}$ to evaluate the evidence for targeted treatments. Five of the 33 DNV-enriched genes (SCN1A, SCN2A, SCN8A, KCNQ2, MECP2) had evidence for therapeutic relevance (CEBM Grade of Recommendation A and B, see Online Methods,

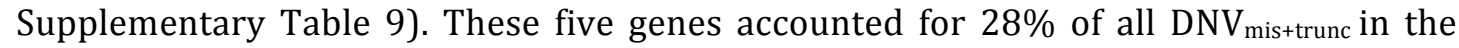
significantly implicated genes. Three additional genes (PTEN, CACNA1A, SLC2A1) with at least two $\mathrm{DNV}_{\text {mistrunc, }}$ which were also known disease genes, also had therapeutic relevance according to CEBM criteria. In total 5\% (84/1587) of DNV misttrunc $_{\text {in NDD with }}$ epilepsy were in genes with therapeutic consequences. According to the guidelines of the American College of Medical Genetics (ACMG,) $)^{36}$ all DNV that are not in ExAC and that are in known disease genes or genes with DNV burden in our dataset are categorized as "likely pathogenic", while we did not apply all ACMG criteria to individual DNV (see online methods).

\section{Comparing DNV between NDD with and without epilepsy}

We compared frequencies of $\mathrm{DNV}_{\text {mistrunc }}$ in NDD with epilepsy (NDD $\mathrm{EE}_{\mathrm{EEE}}$ ) to $\mathrm{NDD}_{\text {woE }}$ across all 107 DNV-enriched genes (logistic regression, see Online Methods). Increasing age at time of recruitment increased likelihood of epilepsy (three-year OR 1.11, 95\%-CI 1.04 to 1.18 , $p$-value $=3 \times 10^{-3}$, individual genes in Supplementary Figure S5). Sex was not associated with epilepsy status ( $p$-value $=0.5$ ). Individuals with $D_{N} V_{\text {mis }}$ were more likely to have epilepsy than individuals with $\mathrm{DNV}_{\text {trunc }}$ (Figure 2, $\mathrm{OR}_{\text {mis }} 2.1,95 \%$-CI 1.6 to 2.8, pvalue $2 \times 10^{-7}$ ). In line with previous reports ${ }^{15}$, we observed this pattern on a single gene level for SCN2A (Firth regression, $\mathrm{OR}_{\mathrm{mis}} 23.5,95 \%$-CI 3.8 to 277 , p-value 0.0003 , Table 1 ). Confirming previous findings $24,37, \mathrm{DNV}$ in ion channel genes were associated with epilepsy (OR 6.0, 95\%-CI 3.9 to 9.2, p-value $\left.1 \times 10^{-16}\right) .83 \%(110 / 133)$ of DNV in ion channel genes were $\mathrm{DNV}_{\text {mis. }}$. However, in the subset of $910 \mathrm{DNV}$ not in ion channel genes, $\mathrm{DNV}_{\text {mis }}$ were still associated with epilepsy (OR 1.5, p-value 0.005, 95\%-CI 1.1 to 2.1), implying that the effect of $\mathrm{DNV}_{\text {mis }}$ on epilepsy was not entirely driven by ion channel genes. We observed a higher rate of $\mathrm{DNV}_{\text {mis }}$ in $\mathrm{NDD}_{\mathrm{EE}}$ than in $\mathrm{NDD}_{\mathrm{uE}}$, though only with nominal significance (Fisher's exact test, OR 1.8, 95\%-CI 1.04 to 3.4, p-value 0.03, Supplementary Figure S10B). Four genes were more frequently mutated in NDD with 
bioRxiv preprint first posted online Apr. 3, 2017; doi: http://dx.doi.org/10.1101/123323. The copyright holder for this preprint

(which was not peer-reviewed) is the author/funder, who has granted bioRxiv a license to display the preprint in perpetuity.

All rights reserved. No reuse allowed without permission.

epilepsy ( $\mathrm{NDD}_{\mathrm{EE}+\mathrm{uE}}$ ) than $\mathrm{NDD}_{\mathrm{woE}}$ (Fisher's Exact Test, Figure 2A/2B, Table 1, Supplementary Table 10). With the exception of $S C N 1 A$, frequencies of DNV were not significantly different per gene between $\mathrm{NDD}_{\mathrm{EE}}$ and $\mathrm{NDD}_{\mathrm{uE}}$ for $\mathrm{DNV}_{\mathrm{mis}}$ or $\mathrm{DNV}_{\text {trunc }}$ (Supplementary Figure S10, Supplementary Table 11).

\section{Evaluation of diagnostic gene panels for epilepsy disorders}

Targeted sequencing of disease-specific gene panels is widely employed in diagnostics of epilepsies ${ }^{18,19}$. We compared our results to 24 diagnostic panels for epilepsy or EE (see Online Methods, full list in Supplementary Table 12). In total, the 24 different panels covered 358 unique genes $(81.5 \pm 8.8$ genes per panel, mean \pm sd). Applying these 24 diagnostic panels on our data set would only have detected on average $59 \%$ of $\mathrm{DNV}_{\text {mistrunc }}$ in the 33 DNV-enriched genes (Supplementary Figure S11). However, similar to most other research studies involving clinical WES7, we cannot fully assess the extent of potential pre-screening. We investigated whether genes in the 24 panels had some evidence for disease association given the following features that we (and others ${ }^{23,33}$ ) observed in genes with DNV burden in NDD: depletion for truncating and missense variants in ExAC ${ }^{32}$ controls as well as brain expression (Online Methods, Supplementary Figure S9). We restricted this analysis to autosomal dominant and X-linked acting panel genes $\left(\mathrm{n}_{\text {dominant+X-linked }}=191\right.$, Supplementary Table 13$) .95 \%(52 / 55)$ of panel genes that had two or more $\mathrm{DNV}_{\text {mistrunc }}$ in our study were both constraint and brain-expressed. However, only $63 \%(86 / 136)$ of panel genes with one or less $\mathrm{DNV}_{\text {mistrunc }}$ in our study were constraint and brain-expressed (Fisher's exact test, OR 10.2, 95\%-CI 3.0 to 53.0, pvalue $2.3 \times 10^{-6}$ ). We applied evidence of disease association as defined by the ClinGen Gene Curation Workgroup 38 , to those 50 panel genes lacking two of the criteria $\mathrm{DNV} /$ brain expression/constraint. We found that ten of the 50 genes had no, eight had limited and seven had conflicting published evidence for disease association (Supplementary Table 14). Thirteen genes showed moderate, strong or definitive evidence for association to entities where neither NDD nor epilepsy were major features which may partly be explained by a panel design containing genes associated with diseases beyond the spectrum of NDD (for further details see Online Methods and Supplementary Figure S11). 


\section{Discussion}

293

294

295

296

297

298

299

300

301

302

303

304

305

306

307

308

309

310

311

312

313

314

315

316

317

318

319

320

321

322

323

324

325

In this study, we systematically investigated DNV in NDD with and without epilepsy. In NDD with epilepsy, we could hardly distinguish individuals ascertained for epileptic encephalopathy and NDD with unspecified epilepsy on a genetic level. Thus, we conclude that these phenotype groups share a spectrum of disease genes predominantly including genes initially reported as EE genes. We identified 33 genes with DNV burden in NDD with epilepsy, of which the majority was expressed in the infant brain and depleted for functional variation in ExAC 32 , as previously described for NDD genes 23,33 . We report statistically robust disease association for SNAP25, GABRB2 and CACNA1E, which was previously lacking (Supplementary Notes).

We found, that individuals with $\mathrm{DNV}_{\text {mis }}$ were generally more likely to have epilepsy than

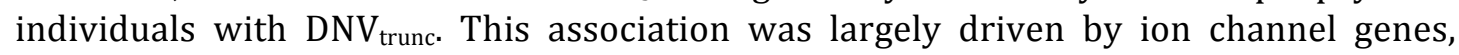
which confirms longstanding statements that many epilepsy disorders act as channelopathies $2,37,24$. Heterozygous $\mathrm{DNV}_{\text {mis }}$ have been shown to cause epilepsy via dominant negative (e.g. $K C N Q 2^{39}$ ) or gain-of-function (e.g. $S C N 8 A^{40}$ ) effects on ion channels. On the individual gene level, missense variants in $S C N 2 A^{15}$ and $S C N 8 A^{41}$ were more strongly implicated in epilepsy than protein truncating variants, which we statistically confirm for $S C N 2 A$. Yet, we found that $\mathrm{DNV}_{\text {mis }}$ were also associated with epilepsy independent of ion channel genes. This may imply that alteration of protein function quantitatively plays a larger role than haploinsufficiency ${ }^{42}$ in the pathophysiology of NDD with epilepsy compared to NDD without epilepsy. We found multiple gene sets enriched for $\mathrm{DNV}_{\text {mis }}$ in epilepsy compared to no epilepsy (see Supplementary Note). The majority was related to ion channels, while others related to neuronal cells (e.g. axon part, synaptic transmission). However, biological interpretation should be done with caution given that previous studies have found that many of these gene sets share a large number of underlying genes ${ }^{22}$ and gene annotations are biased 43 . We further replicate a previous finding that the rate of epilepsy was correlated with severity of intellectual disability $4-6$, implying that brain function could contribute to epileptogenesis or genetic variants cause both epilepsy and NDD. Alternatively, severe epileptic activity may also damage brain function and thereby contribute to NDD, which constitutes the original definition of $\mathrm{EE}^{9,17}$. This is supported by many cases of clinical regression after onset of epilepsy and improvement of NDD through seizure control.

In NDD with epilepsy we found no genetic differences between unspecified epilepsy and EE, with the exception of SCN1A (Supplementary Note). Phenotypic heterogeneity has been described for the majority of EE genes ${ }^{1,11}$, i.e. variants in the same gene could lead to a spectrum of different phenotypes. Due to pleiotropy, individuals that carry a pathogenic DNV in an EE gene and fulfil diagnostic criteria of EE may also be eligible for another NDD diagnosis and thus by chance be assigned to an ASD, DD or ID and not an EE screening cohort. In line with this hypothesis, we found typically EE-associated seizure types (e.g. epileptic spasms) in cohorts with unspecified epilepsy. Some of the diagnostic criteria for $\mathrm{EE}^{1,10}$ may present ambiguously, leading to uncertainty in terminology ${ }^{17}$. Thus, 43\% (21/49) of individuals diagnosed with EE in the Epi4K-E224 study initially presented with DD prior to seizure onset conflicting with the original definition of $\mathrm{EE}^{3,17}$. Clear phenotypic distinction between encephalopathic versus nonencephalopathic epilepsies may therefore be difficult. Accordingly, mechanisms that result in an encephalopathic course of a genetic NDD remain elusive.

Restricting DNA sequencing or DNA sequence analysis to panels of known disease genes is widely used in diagnosis of genetic diseases including epilepsy $(19,100,000$ genomes project [www.genomicsengland.co.uk]). We confirmed that epilepsy gene panels from 
bioRxiv preprint first posted online Apr. 3, 2017; doi: http://dx.doi.org/10.1101/123323. The copyright holder for this preprint

(which was not peer-reviewed) is the author/funder, who has granted bioRxiv a license to display the preprint in perpetuity.

All rights reserved. No reuse allowed without permission.

345 diagnostic laboratories differ substantially in gene content ${ }^{18}$ with at least 25 genes with 346 low evidence for disease association (ClinGen criteria ${ }^{38}$ ). Statistically not robust gene347 disease associations occasionally resulted in false-positive reports of causality posing 348 challenges for correct diagnosis in research and clinical settings ${ }^{11,44}$. Our data provide grounds for replacing genes with limited evidence by genes with higher evidence in the design of gene panels for NDD with epilepsy.

Therapeutic approaches, tailored to the patient's underlying genetic variant, have successfully been applied for several $\mathrm{EE}^{2}$ including treatment with ezogabine in KCNQ2 encephalopathy ${ }^{45}$ or ketogenic diet in $S L C 2 A 1$-related disorders ${ }^{46} .5 \%$ of $\mathrm{DNV}_{\text {mistrunc }}$ in our study were in eight genes (Supplementary Table 9) for which we could confirm therapeutic consequences with established evidence-based medicine criteria ${ }^{35}$. This finding reinforces the urgency of making a genetic diagnosis in NDD with epilepsy. We expect that with increasing understanding of the underlying pathomechanisms, the group of genetic epilepsies with relevant therapeutic consequences will continue to grow. 


\section{Online Methods}

362

363

364

365

366

367

368

369

370

371

372

373

374

375

376

377

378

379

380

381

382

383

384

385

386

387

388

389

390

391

392

393

394

395

396

397

398

399

400

401

402

403

404

405

406

407

408

409

410

411

412

413

\section{Patient cohorts}

For this study, we ascertained 8,529 patients with the following neurodevelopmental disorders (NDD): developmental delay ( $D^{31}, \mathrm{n}=4293$ ), autism spectrum disorder (ASD 20, $n=2508)$, epileptic encephalopathy $24,28 \quad\left(\mathrm{NDD}_{\mathrm{EE}}, \mathrm{n}=529\right)$, intellectual disability $23,25-27$ (ID, n=1035), and epilepsy with $\operatorname{NDD}^{28}(n=164)$. From this cohort, we selected 6753 individuals, for which the presence or absence of epilepsy was ascertained and of whom ca. 88\% had ID (based on assumption of $81.7 \%$ ID in the DDD study $47,89.8 \%$ ID in a diagnostic cohort from AmbryGenetics ${ }^{28}$ and 100\% ID in all other cohorts.) Among individuals with ASD who were phenotyped within the Simon Simplex Consortium ${ }^{29}$, we restricted our analysis to patients with ID (IQ $\left.<70\right)$ as it has been shown that DNV play only a minor role, in normal IQ ASD ${ }^{6,30}$. Previously sequenced trios ( $n=1911$ ), from unaffected siblings of a child with ASD $^{20,29}$, served as control trios. For our main analyses, we stratified this combined cohort of patients with NDD for patients

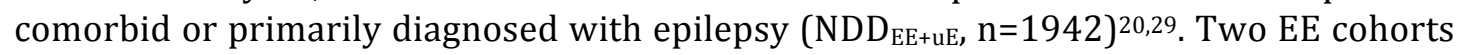
and one ID cohort comprising a combined 144 patients were not previously published; one cohort was only partly published (see Supplementary Table 1). Medical doctors, mostly clinical geneticists, but also neurologists, paediatricians and for ASD ${ }^{29}$ some primary care physicians reported out phenotypes, including presence of epilepsy, in all patients. Our analysis is based on the assumption that medical professionals are sufficiently qualified to diagnose the presence or absence of epilepsy correctly.

\section{Subphenotypes}

We obtained information on specific EE syndromes on $98 \%$ of 518/529 individuals with $\mathrm{NDD}_{\mathrm{EE}}$ (see main text). We obtained specific seizure types (febrile, focal, spasms, generalized) for $55 \%(140 / 256)$ and age of seizure onset for $30 \%(77 / 256)$ of

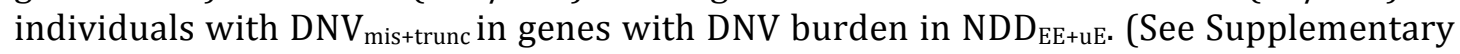
Figure S5 and S6). We did not obtain EEG data per patient. Some patients may have developed epilepsy after inclusion in the study, so we ascertained age at recruitment, that we obtained for $94 \%(1087 / 1157)$ of all individuals with NDD with DNV $_{\text {mistrunc }}$ in DNV-enriched genes (median age at recruitment: 74.8 months). We obtained age of seizure onset for $30 \%(77 / 256)$ of individuals with epilepsy and $\mathrm{DNV}_{\text {misttrunc }}$ in DNVenriched genes (Supplementary Figure S5). We identified 30 individuals with potentially epilepsy-relevant brain malformations (abnormalities of neuronal migration, structural abnormalities of corpus callosum, midbrain, brainstem as schiz-, megal-, holoprosencephaly) in individuals with $\mathrm{DNV}_{\text {misttrunc }}$ in DNV-enriched genes (29 from DDD $^{29}, 1$ from Hamdan et al. ${ }^{7}$ ). 11 of them (37\%) also had seizures.

\section{Whole exome sequencing of parent-patient trios}

In all cohorts, both patients and their unaffected parents underwent whole exome sequencing (WES). Variants that were not present in either parent were considered de novo variants (DNV). 1942 individuals with NDD with epilepsy (NDD $\mathrm{EE}_{\mathrm{EE}}$ ) had 1687 $\mathrm{DNV}_{\text {mis }}$ and $396 \mathrm{DNV}_{\text {trunc }}$ (i.e. stopgain, frameshift, essential splice site). 4811 individuals with $\mathrm{NDD}_{\mathrm{wo}}$ had $4227 \mathrm{DNV}_{\text {mis }}$ and $1120 \mathrm{DNV}_{\text {trunc }}$ (Supplementary Table 2, for individual cohorts see Supplementary Figure S3). The study was approved by the ethics committee of the University of Leipzig (224/16-ek, 402/16-ek) and additional local ethics committees. A list of all published and unpublished cohorts used in this paper can be found in Supplementary Table 1.

Sequencing pipelines of previously unpublished/partly published cohorts (cohorts 8 -11) Libraries were prepared from parents' and patients' DNA, exome captured and sequenced on Illumina sequencers. Raw data was processed and technically filtered 
with established pipelines at the respective academic or diagnostic laboratories. DNV data from all cohorts was re-annotated for this study (see below). Specific pipelines of cohorts 10 to 14 are described below.

Cohort 8 (Ambry Genetics):

419 Diagnostic WES was performed on parent-offspring trios at Ambry Genetics (Aliso Viejo,

420 CA) in 216 individuals with a history of seizures who have been previously described ${ }^{28}$.

421 Genomic DNA extraction, exome library preparation, sequencing, bioinformatics pipeline, and data analyses were performed as previously described ${ }^{48}$. Briefly, samples were prepared and sequenced using paired-end, 100 cycle chemistry on the Illumina HiSeq 2500 sequencer. Exome enrichment was performed using either the SureSelect Target Enrichment System 3.0 (Agilent Technologies) or SeqCap EZ VCRome 2.0 (Roche NimblGen). The sequencing reads were aligned to human reference genome (GRCh37) and variants were called by using CASAVA software (Illumina). The following variants filters were applied to generate a list of high confident de novo variant calls: 1 ) mutation base coverage $>=20 \mathrm{x}$ in all members of the trio; 2 ) heterozygous read ratio in probands $>30 \%$ and $<80 \% ; 3$ ) heterozygous read ratio in parents $<2 \%$; 4 ) genotype quality cutoffs SNV $>100$ and indels $>300$ and 5) exclusion of known sequencing artefacts (based on Ambry Genetics' internal databases).

Cohorts 9 (EuroEPINOMICS RES) and 10 (DFG atypical EE):

Exonic and adjacent intronic sequences were enriched from genomic DNA using the NimbleGen SeqCap EZ Human Exome Library v2.0 enrichment kit. WES was performed using a 100bp paired-end read protocol due to the manufacturer's recommendations on an Illumina HiSeq2000 sequencer by the Cologne Center for Genomics (CCG), Cologne, Germany. Reads were mapped on the human hg19 reference genome (bwa-aln software, bio-bwa.sourceforge.net/). The UnifiedGenotyper (GATK, www.broadinstitute.org/gatk/) and Mpileup (Samtools, http://samtools.sourceforge.net/) software were used to call variants. The paired sample feature from the DeNovoGear software was further used to examine potential de novo mutations in twin pairs. Data analysis and filtering of mapped target sequences was performed with the 'Varbank' exome and genome analysis pipeline v.2.1 (unpublished; https://varbank.ccg.uni-koeln.de). In particular, we filtered for highquality (coverage of more than six reads, fraction of allele carrying reads at least $25 \%$, a minimum genotype quality score of 10, VQSLOD greater than -8) and rare (Caucasian population allele frequency $<0.5 \%$ ) variations on targeted regions + flanking $100 \mathrm{bp}$. In order to exclude pipeline specific artifacts, we also filtered against an in-house cohort of variations, which were created with the same analysis pipeline.

Cohort 11 (University of Leipzig):

Exome capture was carried out with Illumina's Nextera Rapid Capture Exome Kit (Illumina, Inc., San Diego, CA, USA). WES was on an NextSeq500 or HiSeq4000 sequencer (Illumina, Inc.) to $2 \times 150 \mathrm{bp}$ reads at the Centogene AG, Rostock, Germany. Raw sequencing reads were converted to standard fastq format using bcl2fastq software 2.17.1.14 (Illumina, Inc.), and fed to a pipeline at Centogene AG based on the 1000 Genomes Project (1000G) data analysis pipeline and GATK best practice recommendations. Sequencing reads were aligned to the GRCh37 (hg19) build of the human reference genome using bwa-mem (bio-bwa.sourceforge.net/). In addition to GATK HaplotypeCaller (www.broadinstitute.org/gatk/)), variant calling was performed with freebayes (https://github.com/ekg/freebayes) and samtools (http://samtools.sourceforge.net/). Quality filtering of sequencing reads in both parents and children was done according to the following criteria: read depth $>20$, quality $>500$, frequency of alternative allele between 30 and $70 \%$ for the child and not present in the 
parents, frequency $<1 \%$ in internal database, variant called by at least two different genotype callers.

\section{False positive rates of $D N V$}

In cohorts 1 to 4 , all DNV were validated by Sanger sequencing to eliminate false positive calls. In cohorts 5 to 7, through random selection of variants for Sanger validation, the false positive rate was estimated to be approximately $1.4 \%$ and $<5 \%$, respectively. In the clinical cohorts 8 to 11, variants defined as variants worth reporting back to patients (variants of unknown significance or [likely] pathogenic) are normally validated by Sanger sequencing. With this experience, false discovery rates in these cohorts were estimated to be $<5 \%$ (personal communications).

\section{Annotation and Filtering}

DNV files were generated and quality-filtered by the individual groups. All DNV were reannotated with the following pipeline. Variants were annotated with Ensembl's Variant Effect Predictor (http://grch37.ensembl.org/Homo_sapiens/Tools/VEP) of version 82 using database 83 of GRCh37 as reference genome. Per variant, the transcript with the most severe impact, as predicted by VEP, was selected for further analyses. The decreasing order of variant impacts was HIGH, MODERATE, MODIFIER, LOW. Only protein - altering $\mathrm{DNV}\left(\mathrm{DNV}_{\text {mis }}\right.$ or $\mathrm{DNV}_{\text {trunc }}$ [premature stop codon, essential splice site, frameshift]) were included in further analyses. Synonymous DNV ( $\left.D^{2} V_{\text {syn }}\right)$ were analysed as a negative control, as most $\mathrm{DNV}_{\text {syn }}$ have no effect on amino acid sequence in the protein. Variants that were present in $\operatorname{ExAC}^{32}$, an aggregation of 60,706 exome sequences from adult individuals without severe childhood-onset diseases, were excluded after DNV enrichment, as these have been shown to convey no detectable risk to NDD on a group level ${ }^{33}$. For DNV rates per cohort see Supplementary Figure S2. We did not investigate pathogenicity of individual DNV according to the guidelines of the American College of Medical Genetics (ACMG). However, ACMG criteria PS2 (de novo occurrence, with maternity and paternity confirmed) and PM2 (absence from controls) apply to all DNV in our cohort. The combination of PS2 and PM2 classifies a variant as at least "likely pathogenic". ACMG criteria are only applicable to variants in disease associated genes ${ }^{36}$. Therefore, all DNV in known disease genes and genes with genomewide DNV burden in our dataset are presumed likely pathogenic DNV.

\section{Harmonization of different cohorts}

The core analysis of our study is the enrichment of $\mathrm{DNV}_{\text {mistrunc }}$ compared to expectation by a mutational model in individuals with $\mathrm{NDD}_{\mathrm{EE}+\mathrm{nsE}}$. For this analysis, we were conservative in assuming that every gene was well captured across all cohorts. However, when comparing DNV burden across different phenotypes we aimed to separate technical from biological differences with the following methods. In exome sequencing, different capture solutions capture specific exonic regions with different efficiencies. These differences have shown to be quite stable within and across different samples of the same capture kits ${ }^{49}$. We therefore generated a list of exons that displayed consistent high coverage across different capture solutions. We collected published and internal data aiming for the highest possible variety of capture kits using 3,000 samples of 5 different capture kits, including NimbleGen SeqCap v2 and v3, Agilent SureSelect v2, v3, and v5). We generated a list of exons where at least $80 \%$ of all samples had at least $10 \mathrm{x}$ coverage. We excluded the oldest capture kits before calculating the high coverage exons as well as excluding the two oldest cohorts 26,27 from our list of DNV. Restricting to high coverage regions resulted in a loss of ca. $11 \%$ of DNV in DNV-enriched genes. We consequently performed all genotype phenotype comparisons across cohorts (Figures 1A, 2, Supplementary Figures S6-10) with this restricted DNV set. Further, we compared the frequency of $\mathrm{DNV}_{\text {syn }}$ across all cohorts and excluded cohorts of which $\mathrm{DNV}_{\text {syn }}$ were 
not available. In the subset of DNV in high coverage exons, rates of supposedly neutral $\mathrm{DNV}_{\text {syn }}$ were not different between individuals with and without epilepsy (Poisson Exact test, $p$-value $=0.48, \mathrm{RR}=0.99), \mathrm{NDD}_{\mathrm{uE}}$ and $\mathrm{NDD}_{\mathrm{EE}}(\mathrm{p}$-value $=0.65, \mathrm{RR}=0.94)$ or $\mathrm{NDD}$ and controls ( $p$-value $=0.58, R R=0.99$ ). The frequency of $D N V_{\text {mistrunc }}$ was also not different between individuals with and without epilepsy ( $p$-value $=0.5, R R=1.02$ ). Our chances to identify $\mathrm{DNV}_{\text {misttrunc }}$ in EE genes in the epilepsy cohort were therefore not inflated by a higher baseline rate of $\mathrm{DNV}_{\text {mistrunc }}$ in comparison to $\mathrm{NDD}_{\mathrm{woE}}$. We reannotated all DNV in the same way as described above.

\section{Statistical analysis}

All statistical analyses were done with the $\mathrm{R}$ programming language (www.rproject.org). Fisher's Exact Test for Count Data, Wilcoxon rank sum test, Poisson Exact Test, Cochran-Mantel-Haenszel test, logistic regression, Firth regression, Spearman correlation, Welch two-sided t-test and calculation of empirical p-values were performed as referenced in the results. For datasets assumed to be normally distributed after visual inspection, mean and standard deviation (sd) are written as mean \pm sd. When performing Poisson Exact Tests, we reported effect size as rate ratio (RR), which is the quotient of the two rates compared in the test. For Fisher's Exact Test and logistic regression analyses, we reported odds ratios (OR). 95\% confidence intervals were abbreviated as $95 \%$ - $\mathrm{CI}$. The R code used to perform the statistical analyses and figures is available upon request.

\section{DNV enrichment analyses}

To identify genes with a significant DNV burden, we compared numbers of observed with numbers of expected missense, truncating and synonymous DNV per gene using an established framework of gene-specific mutation rates ${ }^{30}$. The analysis was done with the R package denovolyzer ${ }^{50}$, that compares observed versus expected DNV using a Poisson Exact test. We corrected the obtained p-values with the Bonferroni method for the number of genes for which gene specific mutation rates ${ }^{30}$ were available $(n=18225)$ and six tests resulting in a p-value significance threshold of $5 \times 10^{-7}$. Genes that passed that significance threshold for either missense, truncating or both missense plus truncating DNV were considered genes with an exome-wide DNV burden. To compare DNV between disease groups, DNV enrichment analyses were carried out in the cohort of all patients with NDD ( $n=6753)$ as well as in patients with epilepsy ( $\left.N D D_{E E+u E}, n=1942\right)$ and without epilepsy $\left(\mathrm{NDD}_{\mathrm{woE}}, \mathrm{n}=4811\right)$, but only genes with a $\mathrm{DNV}_{\text {misttrunc }}$ burden in the NDD with epilepsy cohort and the combined NDD cohort were reported.

\section{HPO enrichment analyses}

Significantly enriched Human phenotype ontology (HPO) terms were computed with the $\mathrm{R}$ package of g:Profiler ${ }^{34}$, using ordered enrichment analysis on significance-ranked proteins (see Supplementary Table 8). Different gene sets were queried using the background gene set of all 18225 genes for which gene specific mutation rates were available ${ }^{30}$. Only terms that were statistically significant with a Bonferroni corrected pvalue $<0.01$ were reported, as our negative controls (genes with at least two $\mathrm{DNV}_{\text {misttrunc }}$ in healthy control) were not enriched for any functional categories below this p-value.

\section{Therapeutic relevance}

To assess if DNV in our cohort were in genes of therapeutic relevance, we searched the literature for treatment recommendations for all established disease genes with at least two $D N V_{\text {mistrunc }}$ in our NDD with epilepsy cohort. We rated the publications with the standardized score of the Oxford Centre for Evidence-Based Medicine ${ }^{35}$. We only reported and considered genes for which at least one treatment recommendation achieved level of evidence of II or higher. For a list of all genes and levels of evidence see Supplementary Table 9. 
We downloaded the Developmental Transcriptome dataset of 'BrainSpan: Atlas of the Developing Human Brain' (www.brainspan.org, funded by ARRA Awards 1RC2MH089921-01, 1RC2MH090047-01, and 1RC2MH089929-01, 2011). The atlas includes RNA sequencing data generated from tissue samples of developing postmortem brains of neurologically unremarkable donors covering 8 to 16 brain structures. We extracted brain expression data from the 5 donors that were infants aged 0 to 12 months. Per gene, we obtained the median RPKM value of all infant individuals and across brain regions. In all calculations and figures gene expression values are displayed as median $(\log 2+1)$-transformed RPKM values. We defined infant brain gene expression as median $(\log 2+1)$-transformed RPKM value $>1$. More details about tissue acquisition and sequencing methodology can be found in the BrainSpan website's documentation.

\section{Evaluation of genes' intolerance to protein altering variants}

We assessed individual gene tolerance to truncating or missense variants in the general population with the pLI score (probability of being loss-of-function intolerant) and missense z-score. These scores indicate depletion of truncating and missense variants in $\operatorname{ExAC}^{32}(60,706$ individuals without childhood onset diseases), respectively. We used gene constraint cut-offs $>0.9$ for pLI and $>3.09$ for missense-z scores as recommended by the score developers ${ }^{32}$. We calculated empirical p-values to evaluate if pLI scores of exome-wide and nominally DNV-enriched genes were significantly higher compared to pLI scores of random gene sets as described in ${ }^{23}$. Briefly, we computed the expected pLI for a given gene set with size $n$ by randomly drawing 1,000,000 gene sets with size $n$ from the total $18,225 \mathrm{pLI}$ annotated genes. We computed, how many times the median pLI score of randomly sampled gene sets would exceed the median pLI of the gene set under investigation. To that number we added 1 and divided by the number of total samplings +1 to obtain the empirical p-value.

\section{Comparing $D N V$ in $N D D_{E E}, N D D_{u E}$ and $N D D_{\text {woE }}$}

We investigated $\mathrm{DNV}_{\text {mistrunc }}$ in $\mathrm{NDD}_{\mathrm{EE}+\mathrm{uE}+\mathrm{woE}}$ across all 107 genes that were DNVenriched in $\mathrm{NDD}_{\mathrm{EE}+\mathrm{uE}} \mathrm{NDD}_{\mathrm{woE}}$ and/or $\mathrm{NDD}_{\mathrm{EE}+\mathrm{uE}+\mathrm{woE}}$. We restricted our analysis to DNV not in $\mathrm{ExAC}^{23}$ and in high coverage regions. To investigate, if age at time of recruitment, sex or variant class $\left(\mathrm{DNV}_{\text {mis }} / \mathrm{DNV}_{\text {trunc }}\right)$ influenced the presence of epilepsy, we tested them as covariates in a logistic regression model with epilepsy as response variable. We aimed to explore, whether DNV in NDD with epilepsy might be associated with ion channels compared to NDD without epilepsy, as it is a long-established hypothesis, that many epilepsies are channelopathies ${ }^{37}$. We extracted a comprehensive gene set of 237 known ion channel genes from 1766 previously described 22 curated gene sets derived from public pathway databases and publications (see Supplementary Note). To investigate if ion channel genes were associated with epilepsy we included annotation as ion channel gene as a categorical predictor in the logistic regression model. We used Firth regression to assess the effect of variant class on the presence of epilepsy for individual genes. We used Fisher's Exact test to compare frequencies of DNV per gene between phenotype groups. To account for multiple testing, we corrected p-values for the number of tests performed (Bonferroni method).

\section{Diagnostic gene panels for epileptic encephalopathy/comprehensive epilepsy from 24 academic/commercial providers}

We set out to compare our results to diagnostic gene panels for epileptic encephalopathy of international commercial and academic providers. We searched the Genetic Testing Registry (GTR) ${ }^{51}$ of NCBI (date: 01/2017) for providers of tests for "Epileptic encephalopathy, childhood-onset" and identified 16 diagnostic epilepsy 
panels. We excluded 3 panels with $<20$ or $>200$ genes and added 11 additional diagnostic providers not registered at GTR to evaluate 24 diagnostic panels targeting epilepsy in general $(n=11)$ or EE specifically $(n=13)$. The gene content covered in each of the 24 gene panels can be found in Supplementary Table 11. Gene lists were freely available for download at the respective providers' websites. For each of the 33 genes with DNV burden in NDD with epilepsy, we calculated to what proportion they were included in 24 commercial or academic providers of gene panels for epileptic encephalopathy/comprehensive epilepsy. For each gene, we then multiplied the percentage of inclusion in any of the 24 panels by the total number of $\mathrm{DNV}_{\text {mist+trunc }}$ of that gene in the cohort of 1942 individuals with $\mathrm{NDD}_{\mathrm{EE}+\mathrm{uE}}$. We investigated if there were genes in the 24 diagnostic gene panels without evidence for implication in NDD with epilepsy. We focused on 191 dominant or X-linked panel genes (listed in Supplementary Table 14). We tested these genes for three criteria of association with NDD with epilepsy: Firstly, if genes had at least two $D N V_{\text {mistrunc }}$ in our study; secondly, whether genes were expressed in the infant brain defined by a median RPKM of all samples and brain regions $>1$; thirdly, whether genes had a pLI $>0.9$ or missense z-score $>3.09$ indicating intolerance to truncating or missense variants ${ }^{32}$. We intersected these lists to nominate genes that did not display features of DNV-enriched genes in this study. On these genes we applied ClinGen criteria ${ }^{38}$ for gene-disease association.

\section{Data availability}

The authors declare that all data used for computing results supporting the findings of this study are available within the paper and its supplementary information files. Raw sequencing data of published cohorts are referenced at the respective publications. Raw sequencing data of cohort EuroEPINOMICS RES have been deposited in the European Genome-phenome Archive (EGA) with the accession code EGAS00001000048 (https://www.ebi.ac.uk/ega/datasets/EGAD00001000021). Raw sequencing data of cohort 10 (DFG atypical EE) will be deposited in a public repository after finalization of 
bioRxiv preprint first posted online Apr. 3, 2017; doi: http://dx.doi.org/10.1101/123323. The copyright holder for this preprint (which was not peer-reviewed) is the author/funder, who has granted bioRxiv a license to display the preprint in perpetuity.

All rights reserved. No reuse allowed without permission.

Figure 1. $\mathrm{DNV}_{\text {mistrunc }}$ in EE genes in different cohorts of NDD. A, The proportion of $\mathrm{DNV}_{\text {mistrunc }}$ in EE genes is not significantly different between patients with $\mathrm{NDD}_{\mathrm{EE}}$ (red) and $\mathrm{NDD}_{\mathrm{uE}}$ (blue), but higher than $\mathrm{NDD}_{\mathrm{woE}}$ (yellow) or healthy controls (grey). Cohort size is given as number of individuals (Ind). B, Proportion of DNV in EE genes in patients with versus without epilepsy across different NDD (DD, ASD, ID). P-values are plotted next to respective odds ratios (red dots), while 95\%-CI are shown as yellow bars (Fisher's exact test for individual cohorts, Cochran-Mantel-Haenszel test for combined

A,

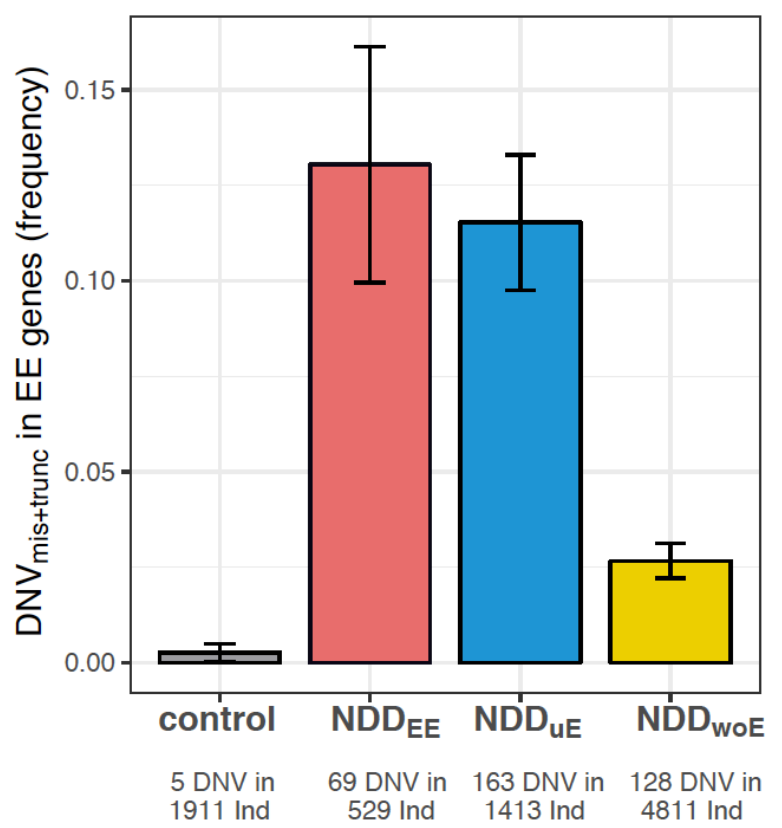

673

674 B,

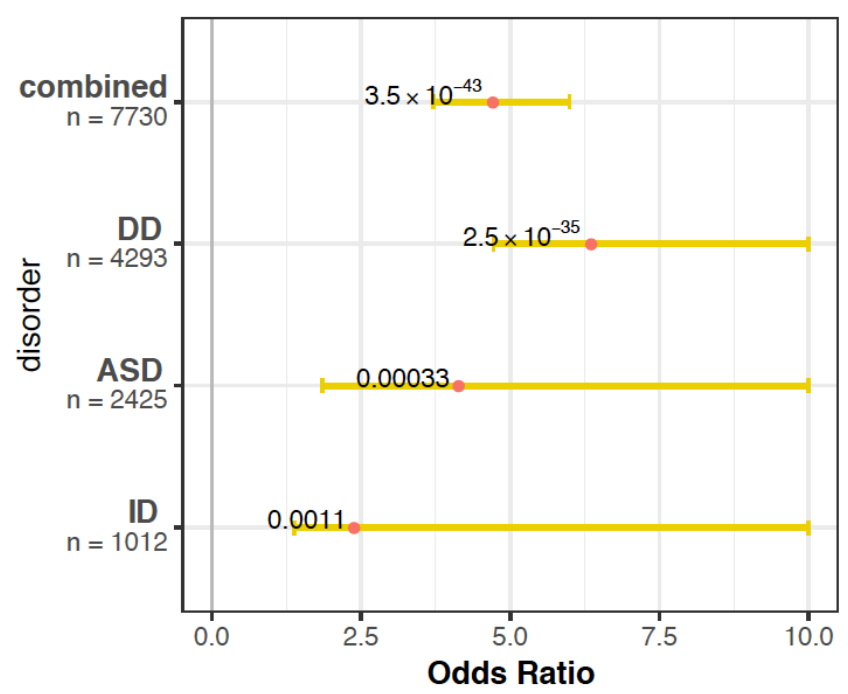

DNV in EE genes, epilepsy vs. no epilepsy 
bioRxiv preprint first posted online Apr. 3, 2017; doi: http://dx.doi.org/10.1101/123323. The copyright holder for this preprint (which was not peer-reviewed) is the author/funder, who has granted bioRxiv a license to display the preprint in perpetuity.

All rights reserved. No reuse allowed without permission.

676

677

678

679

680

681

682

A,

683

684

B,

Figure 2. DNV in patients with epilepsy (NDD $D_{E E+u E}$ ) versus without epilepsy (NDD $\left.D_{w o E}\right)$ in 107 genes with significant $\mathrm{DNV}$ burden. $\mathbf{A}, \mathrm{DNV}_{\text {mis, }} \mathbf{B}, \mathrm{DNV}_{\text {trunc. }}$ Genes with different frequencies are labeled (method: Fisher's Exact test, blue: nominal significance, p-value $<0.05$, red: significant after correcting for 266 tests). The dotted line represents equal frequency of DNV in NDD with and without epilepsy.
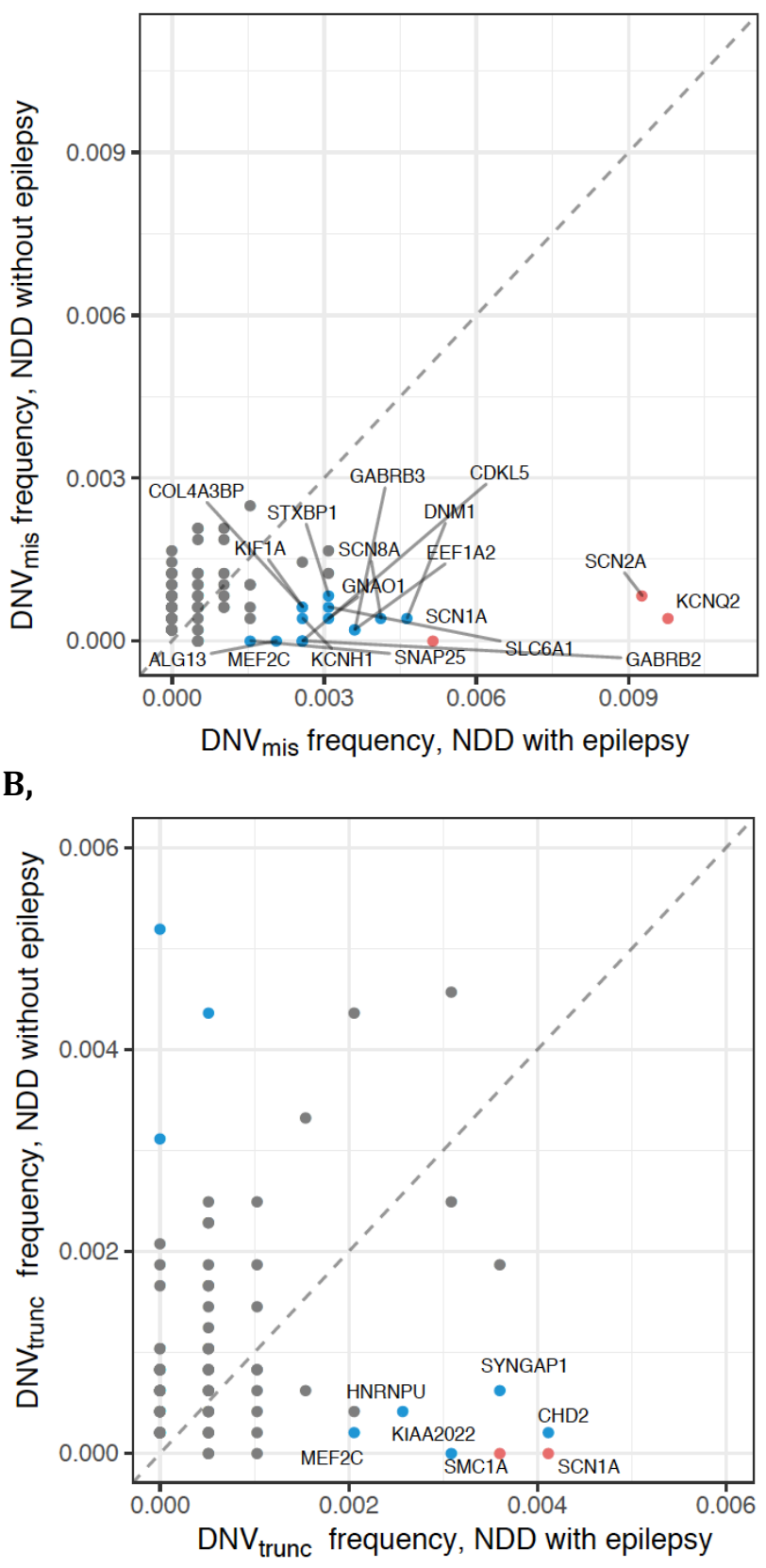
bioRxiv preprint first posted online Apr. 3, 2017; doi: http://dx.doi.org/10.1101/123323. The copyright holder for this preprint (which was not peer-reviewed) is the author/funder, who has granted bioRxiv a license to display the preprint in perpetuity.

\section{References}

1. McTague, A., Howell, K.B., Cross, J.H., Kurian, M.A. \& Scheffer, I.E. The genetic landscape of the epileptic encephalopathies of infancy and childhood. Lancet Neurol 15, 304-16 (2016).

2. Myers, C.T. \& Mefford, H.C. Advancing epilepsy genetics in the genomic era. Genome Med 7, 91 (2015).

3. Scheffer, I.E. et al. ILAE classification of the epilepsies: Position paper of the ILAE Commission for Classification and Terminology. Epilepsia (2017).

4. McGrother, C.W. et al. Epilepsy in adults with intellectual disabilities: prevalence, associations and service implications. Seizure 15, 376-86 (2006).

5. Thomas, S., Hovinga, M.E., Rai, D. \& Lee, B.K. Brief Report: Prevalence of Co-occurring Epilepsy and Autism Spectrum Disorder: The U.S. National Survey of Children's Health 2011-2012. J Autism Dev Disord 47, 224-229 (2017).

6. Robinson, E.B. et al. Autism spectrum disorder severity reflects the average contribution of de novo and familial influences. Proc Natl Acad Sci U S A 111, 15161-5 (2014).

7. Deciphering Developmental Disorders, S. Prevalence and architecture of de novo mutations in developmental disorders. Nature 542, 433-438 (2017).

8. Li, J. et al. Genes with de novo mutations are shared by four neuropsychiatric disorders discovered from NPdenovo database. $\mathrm{Mol}$ Psychiatry 21, 290-7 (2016).

9. Berg, A.T. et al. Revised terminology and concepts for organization of seizures and epilepsies: report of the ILAE Commission on Classification and Terminology, 2005-2009. Epilepsia 51, 676-85 (2010).

10. Berg, A.T. \& Millichap, J.J. The 2010 revised classification of seizures and epilepsy. Continuum (Minneap Minn) 19, 571-97 (2013).

11. Helbig, I. \& Tayoun, A.A. Understanding Genotypes and Phenotypes in Epileptic Encephalopathies. Mol Syndromol 7, 172-181 (2016).

12. Trump, N. et al. Improving diagnosis and broadening the phenotypes in early-onset seizure and severe developmental delay disorders through gene panel analysis. J Med Genet 53, 310-7 (2016).

13. Depienne, C. et al. Spectrum of SCN1A gene mutations associated with Dravet syndrome: analysis of 333 patients. J Med Genet 46, 183-91 (2009).

14. Weckhuysen, S. et al. Extending the KCNQ2 encephalopathy spectrum: clinical and neuroimaging findings in 17 patients. Neurology 81, 1697-703 (2013).

15. Wolff, M. et al. Genetic and phenotypic heterogeneity suggest therapeutic implications in SCN2A-related disorders. Brain (2017).

16. Stamberger, H. et al. STXBP1 encephalopathy: A neurodevelopmental disorder including epilepsy. Neurology 86, 954-62 (2016).

17. Howell, K.B., Harvey, A.S. \& Archer, J.S. Epileptic encephalopathy: Use and misuse of a clinically and conceptually important concept. Epilepsia 57, 343-7 (2016).

18. Chambers, C., Jansen, L.A. \& Dhamija, R. Review of Commercially Available Epilepsy Genetic Panels. J Genet Couns 25, 213-7 (2016). 
736

737

738

739

740

741

742

743

744

745

746

747

748

749

750

751

752

753

754

755

756

757

758

759

760

761

762

763

764

765

766

767

768

769

770

771

772

773

774

775

776

777

778

779

780

781

782

19. Lemke, J.R. et al. Targeted next generation sequencing as a diagnostic tool in epileptic disorders. Epilepsia 53, 1387-98 (2012).

20. Iossifov, I. et al. The contribution of de novo coding mutations to autism spectrum disorder. Nature 515, 216-21 (2014).

21. Zaidi, S. et al. De novo mutations in histone-modifying genes in congenital heart disease. Nature 498, 220-3 (2013).

22. Singh, T. et al. The contribution of rare variants to risk of schizophrenia in individuals with and without intellectual disability. Nat Genet (2017).

23. Lelieveld, S.H. et al. Meta-analysis of 2,104 trios provides support for 10 new genes for intellectual disability. Nat Neurosci 19, 1194-6 (2016).

24. EuroEpinomics \& Epi4K. De novo mutations in synaptic transmission genes including DNM1 cause epileptic encephalopathies. Am J Hum Genet 95, 360-70 (2014).

25. Hamdan, F.F. et al. De novo mutations in moderate or severe intellectual disability. PLoS Genet 10, e1004772 (2014).

26. de Ligt, J. et al. Diagnostic exome sequencing in persons with severe intellectual disability. $N$ Engl J Med 367, 1921-9 (2012).

27. Rauch, A. et al. Range of genetic mutations associated with severe nonsyndromic sporadic intellectual disability: an exome sequencing study. Lancet 380, 1674-82 (2012).

28. Helbig, K.L. et al. Diagnostic exome sequencing provides a molecular diagnosis for a significant proportion of patients with epilepsy. Genet Med 18, 898-905 (2016).

29. Fischbach, G.D. \& Lord, C. The Simons Simplex Collection: a resource for identification of autism genetic risk factors. Neuron 68, 192-5 (2010).

30. Samocha, K.E. et al. A framework for the interpretation of de novo mutation in human disease. Nat Genet 46, 944-50 (2014).

31. McRae, J.F. et al. Prevalence, phenotype and architecture of developmental disorders caused by de novo mutation. bioRxiv (2016).

32. Lek, M. et al. Analysis of protein-coding genetic variation in 60,706 humans. Nature 536, 285-91 (2016).

33. Kosmicki, J.A. et al. Refining the role of de novo protein-truncating variants in neurodevelopmental disorders by using population reference samples. Nat Genet (2017).

34. Reimand, J. et al. g:Profiler-a web server for functional interpretation of gene lists (2016 update). Nucleic Acids Res 44, W83-9 (2016).

35. OCEBM Levels of Evidence Working Group* (Jeremy Howick, I.C.J.L.L., Paul Glasziou, Trish Greenhalgh, Carl Heneghan, Alessandro Liberati, Ivan Moschetti, Bob Phillips, Hazel Thornton, Olive Goddard and Mary Hodgkinson). The Oxford Levels of Evidence. in Oxford Centre for Evidence-Based Medicine. (2009).

36. Richards, S. et al. Standards and guidelines for the interpretation of sequence variants: a joint consensus recommendation of the American College of Medical Genetics and Genomics and the Association for Molecular Pathology. Genet Med 17, 405-24 (2015).

37. Mulley, J.C., Scheffer, I.E., Petrou, S. \& Berkovic, S.F. Channelopathies as a genetic cause of epilepsy. Curr Opin Neurol 16, 171-6 (2003). 
bioRxiv preprint first posted online Apr. 3, 2017; doi: http://dx.doi.org/10.1101/123323. The copyright holder for this preprint

(which was not peer-reviewed) is the author/funder, who has granted bioRxiv a license to display the preprint in perpetuity.

All rights reserved. No reuse allowed without permission.

783

784

785

786

787

788

789

790

791

792

793

794

795

796

797

798

799

800

801

802

803

804

805

806

807

808

809

810

811

812

813

814

815

816

817

818

38. Rehm, H.L. et al. ClinGen--the Clinical Genome Resource, www.clinicalgenome.org/site/assets/files/2657/current_clinical_validity _classifications.pdf. $N$ Engl J Med 372, 2235-42 (2015).

39. Weckhuysen, S. et al. KCNQ2 encephalopathy: emerging phenotype of a neonatal epileptic encephalopathy. Ann Neurol 71, 15-25 (2012).

40. Wagnon, J.L. et al. Pathogenic mechanism of recurrent mutations of SCN8A in epileptic encephalopathy. Ann Clin Transl Neurol 3, 114-23 (2016).

41. Blanchard, M.G. et al. De novo gain-of-function and loss-of-function mutations of SCN8A in patients with intellectual disabilities and epilepsy. J Med Genet 52, 330-7 (2015).

42. Wilkie, A.0. The molecular basis of genetic dominance. J Med Genet 31, 8998 (1994).

43. Haynes, W., Tomczak, A. \& Khatri, P. Gene annotation bias impedes biomedical research. bioRxiv (2017).

44. MacArthur, D.G. et al. Guidelines for investigating causality of sequence variants in human disease. Nature 508, 469-76 (2014).

45. Millichap, J.J. et al. KCNQ2 encephalopathy: Features, mutational hot spots, and ezogabine treatment of 11 patients. Neurol Genet 2, e96 (2016).

46. De Giorgis, V. \& Veggiotti, P. GLUT1 deficiency syndrome 2013: current state of the art. Seizure 22, 803-11 (2013).

47. Study, D.D.D. Prevalence and architecture of de novo mutations in developmental disorders. Nature 542, 433-438 (2017).

48. Farwell, K.D. et al. Enhanced utility of family-centered diagnostic exome sequencing with inheritance model-based analysis: results from 500 unselected families with undiagnosed genetic conditions. Genet Med 17, 578-86 (2015).

49. Chilamakuri, C.S. et al. Performance comparison of four exome capture systems for deep sequencing. BMC Genomics 15, 449 (2014).

50. Ware, J.S., Samocha, K.E., Homsy, J. \& Daly, M.J. Interpreting de novo Variation in Human Disease Using denovolyzeR. Curr Protoc Hum Genet 87, 725 1-15 (2015).

51. Rubinstein, W.S. et al. The NIH genetic testing registry: a new, centralized database of genetic tests to enable access to comprehensive information and improve transparency. Nucleic Acids Res 41, D925-35 (2013). 